\title{
THE KERNEL OF INTEGRAL CUP PRODUCT
}

\author{
JONATHAN A. HILLMAN \\ (Received 3 January 1986; revised 17 March 1986) \\ Communicated by J. H. Rubinstein
}

\begin{abstract}
We compute the kernel of cup product of 1-dimensional cohomology classes for a group $G$ acting trivially on $\mathbf{Z}$ or $\mathbf{F}_{2}$, by means of the naturality of cup product and the 5-term exact sequence of low degree of a suitable LHS spectral sequence. We determine thereby when cup product is injective, and when it is null.

1980 Mathematics subject classification (Amer. Math. Soc.): 20 J 05.

Keywords and phrases: cohomology, cup product, LHS spectral sequence.

We shall relate the kernel of cup production of 1-dimension cohomology classes for a finitely generated group $G$ acting trivially on $R=\mathbb{Z}$ or $\mathbb{F}_{2}$ to the dual of the second stage of a descending central series for $G$. In [2] we used explicit calculations with cochains to compute this kernel when $E=\mathbf{Q}, \mathbb{F}_{2}$ or $\mathbb{F}_{p}$; here we shall use instead the naturality of cup product and the exact sequence of low degree for an LHS spectral sequence to reduce the case when $G=R^{b}$ for some $b$.

Let $G_{n}$ be the $n$th term of the lower central series for $G$ (defined inductively by $G_{1}=G$ and $\left.G_{n+1}=\left[G, G_{n}\right]\right)$ and let $X^{k}(G)$ be the verbal subgroup generated by all $k$ th powers of elements of $G$. If $R$ is a ring let $G(R)=\cap k e r \lambda$, where the intersection is taken over all homomorphisms $\lambda$ of $G$ into the underlying additive group of $R$. Then we shall show
\end{abstract}

THEOREM 1. Cup product determines a homomorphism from $H^{1}(G ; \mathbb{Z}) \wedge H^{1}(G ; \mathbb{Z})$ to $H^{2}(G ; \mathbb{Z})$ with kernel naturally isomorphic to $\operatorname{Hom}(G(\mathbb{Z}) /[G, G(\mathbb{Z})], \mathbb{Z})$.

(c) 1987 Australian Mathematical Society $0263-6115 / 87 \$ A 2.00+0.00$ 
CoROllary $[3,5,6]$. The kernel of cup product with coefficients $\mathbf{Q}$ is isomorphic to $\operatorname{Hom}\left(G_{2} / G_{3}, \mathbf{Q}\right)$.

THEOREM 2. Cup product determines a homomorphism from $H^{1}\left(G ; \mathbb{F}_{2}\right)$ $\odot H^{1}\left(G ; \mathbb{F}_{2}\right)$ to $H^{2}\left(G ; \mathbb{F}_{2}\right)$ with kernel naturally isomorphic to $\operatorname{Hom}\left(X^{2}(G) /\left[G, X^{2}(G)\right] X^{4}(G), \mathbb{F}_{2}\right)$.

When our argument is applied in the case $R=\mathbb{F}_{p}$ with $p$ odd, we obtain easily an "upper bound" for the kernel, but to recover the full result of [2] we are faced with cochain calculations seemingly worse than those of that paper, and we shall merely quote its result, that cup product from $H^{1}\left(G ; \mathbb{F}_{p}\right) \wedge H^{1}\left(G ; \mathbb{F}_{p}\right)$ to $H^{2}\left(G ; \mathbb{F}_{p}\right)$ has kernel isomorphic to $\operatorname{Hom}\left(G_{2} X^{p}(G) / G_{3} X^{p}(G), \mathbb{F}_{p}\right)$.

As applications of our main results, we determine when cup product (with coefficients $\mathbf{Z}, \mathbb{Q}$ or $\mathbb{F}_{p}$ ) is injective, and when it is null. In particular if $G_{2}=G_{3}$ (for example, if $G$ is abelian) then cup product with coefficients $\mathbb{Z}, \mathbb{Q}$ or $\mathbb{F}_{p}$ ( $p$ odd) is always injective; likewise for $\mathbb{F}_{2}$ if $G$ does not map onto $\mathbb{Z} / 4 \mathbb{Z}$. If $G / G_{3}$ is free nilpotent of class 2 then cup product with any (trivial) coefficients is null. (Wood [6] obtains sufficient conditions for injectivity with these coefficients, in the more general situation of cup product of $k \geqslant 2$ classes of degree $n \geqslant 1$. However his assertion for coefficients $\mathbb{F}_{2}$ is wrong, even for $G=\mathbb{Z} / 4 \mathbb{Z}$. Dwyer [1] relates nullity to relative freeness of certain nilpotent quotients of $G$.)

In Section 1 we describe the cup product homomorphism and give an exact sequence containing the kernel of cup product. The argument is essentially the same for each choice of coefficients. In the later sections we apply this exact sequence to the cases $\mathbf{Z}$ (and $\mathbf{Q}$ ), $\mathbb{F}_{2}$ and $\mathbb{F}_{p}$ ( $p$ odd) in turn.

\section{An exact sequence}

Recall that the low dimensional cohomology groups of $G$ with coefficients in the trivial $G$-module $R$ are $H^{0}(G ; R)=R, H^{1}(G ; R)=\operatorname{Hom}(G, R)$ and $H^{2}(G ; R)=\left\{F: G^{2} \rightarrow R \mid F(h, j)-F(g h, j)+F(g, h j)-F(g, h)=0\right.$ for all $g, h, j$ in $G\} / B$ where $B=\{\partial f:\langle g, h\rangle \rightarrow f(g)+f(h)-f(g h)$ for all $g, h$ in $G \mid f: G \rightarrow R\}$. (We shall henceforth abbreviate $H^{*}(G ; R)$ as $H^{*}(G)$.) The cup product of two elements $f_{1}, f_{2}$ in $H^{1}(G)$ is represented by the function $f_{1} f_{2}$ : $\langle g, h\rangle \rightarrow f_{1}(g) f_{2}(h)$ for all $g, h$ in $G$. Since $f_{1}(g) f_{2}(h)+f_{2}(g) f_{1}(h)=$ $-f_{1}(g) f_{2}(g)-f_{1}(h) f_{2}(h)-\left(-f_{1}(g h) f_{2}(g h)\right)$, cup product $U: H^{1}(G) \times H^{1}(G) \rightarrow$ $H^{2}(G)$ is anticommutative $\left(f_{1} \cup f_{2}=-f_{2} \cup f_{1}\right)$ and so induces a homomorphism $D\left(H^{1}(G)\right) \rightarrow H^{2}(G)$, where $D(M)=M \otimes_{R} M /\langle m \otimes n+n \otimes m| m, n$ in $\left.M\right\rangle$ for any $R$-module $M$. If 2 is invertible in $R$, in particular if $R$ is a field of characteristic $\neq 2$, then $D(M)=M \wedge M$ (the alternating product). This is 
also the case if $R=\mathbf{Z}$. For if $f$ is a homomorphism: $G \rightarrow \mathbb{Z}$ then $F(h)=$ $f(h)(1-f(h)) / 2$ for all $h$ in $G$ defines a function $F: G \rightarrow \mathbb{Z}$ such that $F(g)+$ $F(h)-F(g h)=f(g) f(h)$ for all $g, h$ in $G$, so $f \cup f=0$. Thus if $R=\mathbb{Z}$ or $\mathbb{F}_{p}$ with $p$ odd, cup product gives rise to a $R$-homomorphism (which we shall also call cup product) from $H^{1}(G) \wedge H^{1}(G)$ to $H^{2}(G)$. If $R=\mathbb{F}_{2}$ then $D(M)=M \odot M$ (the symmetric product) and so there is a cup product homomorphism from $H^{1}\left(G ; \mathbb{F}_{2}\right) \odot H^{1}\left(G ; \mathbb{F}_{2}\right)$ to $H^{2}\left(G ; \mathbb{F}_{2}\right)$.

Let $G=G / G(R)$. Then the epimorphism $G \rightarrow \bar{G}$ induces an isomorphism $H^{1}(\bar{G}) \rightarrow H^{1}(G)$. Moreover if $R=\mathbf{Z}$ or $\mathbb{F}_{p}$, and if $G$ (or merely $\bar{G}$ ) is finitely generated, then $\bar{G}$ is isomorphic to $R^{b}$ for some $b$. The 5-term exact sequence of low degree for the LHS cohomology spectral sequence of the extension $1 \rightarrow G(R)$ $\rightarrow G \rightarrow \bar{G} \rightarrow 1$ then gives an exact sequence $0 \rightarrow H^{0}\left(\bar{G} ; H^{1}(G(R))\right) \stackrel{\tau}{\rightarrow}$ $H^{2}(\bar{G}) \rightarrow H^{2}(G)$, where $\tau$ is the transgression homomorphism. (Note that the $\bar{G}$-module $H^{1}(G(R))$ is in general nontrivial, and that $H^{0}\left(\bar{G} ; H^{1}(G(R))\right) \approx$ $\operatorname{Hom}(G(R) /[G, G(R)], R)$.)

If $R=\mathbf{Z}$ or $\mathbb{F}_{p}$ ( $p$ odd) we then have a commutative diagram

$$
\begin{aligned}
0 & \rightarrow 0 \longrightarrow H^{1}(\bar{G}) \wedge H^{1}(\bar{G}) \stackrel{\approx}{\rightarrow} H^{1}(G) \wedge H^{1}(G) \rightarrow 0 \\
& \downarrow \\
0 & \rightarrow H^{0}\left(\bar{G} ; H^{1}(G(R))\right) \stackrel{\tau}{\longrightarrow} H^{2}(\bar{G}) \longrightarrow U_{\bar{G}} \longrightarrow H^{2}(G)
\end{aligned}
$$

where the (right hand) vertical maps are given by cup product. For $R=\mathbb{F}_{2}$ we have a similar diagram, using instead the symmetric product. The snake-lemma then gives us an exact sequence

$$
0 \rightarrow \operatorname{ker}\left(U_{\bar{G}}\right) \rightarrow \operatorname{ker}\left(U_{G}\right) \rightarrow H^{0}\left(\bar{G} ; H^{1}(G(R))\right) \rightarrow \operatorname{coker}\left(U_{\bar{G}}\right) \rightarrow \operatorname{coker}\left(U_{G}\right) .
$$

\section{Coefficients $\mathbb{Z}$ and $\mathbb{Q}$}

Suppose first that $R=\mathbf{Z}$. Then $\bar{G}$ is free abelian, $\bar{G} \approx \mathbb{Z}^{b}$ say, and $H^{*}(\bar{G} ; \mathbb{Z})$ is the exterior algebra generated by $H^{1}(\bar{G} ; \mathbb{Z})$. (For by the Künneth theorem $H^{*}(\bar{G} ; \mathbf{Z})$ is the (graded commutative) $b$-fold tensor product of copies of $H^{1}(\mathbf{Z} ; \mathbf{Z})$, which in turn is isomorphic to $\mathbb{Z}[x] /\left(x^{2}\right)$, where $x=\mathrm{id}_{\mathbf{Z}}$ generates $H^{1}(\mathbf{Z} ; \mathbf{Z})$.) In particular $\operatorname{ker}\left(U_{\bar{G}}\right)=0=\operatorname{coker}\left(U_{\bar{G}}\right)$ and so by the exact sequence above $\operatorname{ker}\left(U_{G}\right)=H^{0}\left(\bar{G} ; H^{\mathbf{l}}(G(\mathbf{Z}))\right)=\left\{f\right.$ in $\operatorname{Hom}(G(\mathbf{Z}), \mathbf{Z}) \mid f\left(g h g^{-1}\right)=f(h)$ for all $g$ in $G$ and $h$ in $G(\mathbf{Z})\}=\operatorname{Hom}(G(\mathbf{Z}) /[G, G(\mathbf{Z})], \mathbf{Z})$. This proves Theorem 1 .

Let $G_{2}=[G, G]$ and $G_{3}=\left[G, G_{2}\right]$. Since $G / G_{2}$ is finitely generated, so is $G_{2} / G_{3}$, and the inclusion of $G_{2}$ into $G(Z)$ induces a map of abelian groups: $G_{2} / G_{3} \rightarrow G(\mathbf{Z}) /[G, G(\mathbf{Z})]$ which has finite kernel and cokernel. Since $Q$ is a flat $\mathbf{Z}$-module, we may then identify the kernel of cup product with coefficients $\mathbf{Q}$ 
with $\operatorname{Hom}\left(G_{2} / G_{3}, \mathbb{Q}\right)$, giving the Corollary. (This result was given by Sullivan in [5]. He dualized the skew symmetric commutator pairing: $G / G_{2} \times G / G_{2} \rightarrow G_{3}$, and this idea was used in [2] for all prime fields.) In particular cup product with coefficients $\mathbf{Z}$ or $\mathbb{Q}$ is injective if and only if $G_{2} / G_{3}$ is finite.

On the other hand, let $\theta$ be a map from a free group $F$ to $G$ which induces an isomorphism $\theta^{*}: H^{1}(G ; \mathbf{Z}) \rightarrow H^{1}(F ; \mathbf{Z})$. Then cup product with coefficients $R=\mathbf{Z}$ or $\mathbb{Q}$ is identically 0 if and only if the induced map from $\operatorname{Hom}(G(R) /[G, G(R)], R)$ to $\operatorname{Hom}\left(F_{2} / F_{3}, R\right)$ is an isomorphism. (For there is a commutative diagram

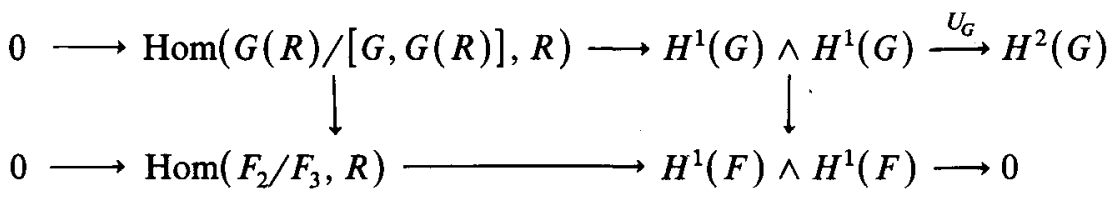

where the right hand vertical map is an isomorphism by assumption and the lower horizontal map is an isomorphism since $H^{2}(F)=0$.) In particular if $R=\mathbf{Z}$ and $G / G_{2}$ is torsion free or if $R=\mathbf{Q}$ this is so if and only if the induced map $\theta_{3}$ : $F / F_{3} \rightarrow G / G_{3}$ is a monomorphism. (See also Proposition 4.3 of [1], which more generally relates the vanishing of higher Massey products to the relative freeness of the nilpotent quotients $G / G_{n}$.)

\section{Coefficients $\mathbb{F}_{2}$}

Suppose next that $R=\mathbb{F}_{2}$. Then $G(R)=X^{2}(G)$, and $\bar{G} \approx(\mathbb{Z} / 2 \mathbb{Z})^{r}$ say. We may again compute $H^{*}(\bar{G})$ via the Künneth theorem. Since $H^{*}(\mathbb{Z} / 2 \mathbb{Z})=\mathbb{F}_{2}[w]$ where $w=\mathrm{id}_{\mathbf{Z} / 2 \mathbf{Z}}$ generates $H^{1}(\mathbf{Z} / 2 \mathbb{Z})$, the cohomology ring of $\bar{G}$ is the polynomial algebra generated in degree 1 by $H^{1}(\bar{G})$. In particular $U_{\bar{G}}: H^{1}(\bar{G}) \odot H^{1}(\bar{G})$ $\rightarrow H^{2}(\bar{G})$ is an isomorphism, and so $\operatorname{ker}\left(U_{G}\right)=\operatorname{Hom}\left(X^{2}(G) /\left[G, X^{2}(G)\right], \mathbb{F}_{2}\right)=$ $\operatorname{Hom}\left(X^{2}(G) /\left[G, X^{2}(G)\right] \cdot X^{4}(G), \mathbb{F}_{2}\right)$. This proves Theorem 2 .

To relate this to the more complicated description of $\operatorname{ker}\left(U_{G}\right)$ given in [2], note that the $\mathbb{F}_{2}$-vector space $X^{2}(G) /\left[G, X^{2}(G)\right] \cdot X^{4}(G)$ contains $G_{2} X^{4}(G) /\left[G, X^{2}(G)\right] \cdot X^{4}(G)$ as a subspace, with quotient $X^{2}(G) / G_{2} X^{4}(G)$. Therefore $\operatorname{ker}\left(U_{G}\right)$ maps onto $\operatorname{Hom}\left(G_{2} X^{4}(G) /\left[G, X^{2}(G)\right] \cdot X^{4}(G), \mathbb{F}_{2}\right)$, with kernel $\operatorname{Hom}\left(X^{2}(G) / G_{2} X^{4}(G), \mathbb{F}_{2}\right)$. Now let $A=G / G_{2} X^{4}(G)$, so $A$ is an abelian group of exponent 4 , with subgroup $2 A=X^{2}(A) \approx X^{2}(G) / G_{2} X^{4}(G)$. Then restriction induces a homomorphism from $\operatorname{Hom}(A, \mathbb{Z} / \mathbf{4 Z})$ to $\operatorname{Hom}(2 A, 2 \mathbf{Z} / 4 \mathbb{Z})$ $\approx \operatorname{Hom}\left(2 A, \mathbb{F}_{2}\right)$ which is easily seen to be onto, and whose kernel is the kernel of reduction modulo (2), $\rho: \operatorname{Hom}(A, \mathbf{Z} / 4 \mathbf{Z}) \rightarrow \operatorname{Hom}\left(A, \mathbb{F}_{2}\right)$. Therefore $\operatorname{Hom}\left(X^{2}(G) / G_{2} X^{4}(G), \mathbb{F}_{2}\right)$ is isomorphic to the image of $\operatorname{Hom}(G, \mathbf{Z} / 4 \mathbb{Z}) \approx$ $\operatorname{Hom}(A, \mathbf{Z} / 4 \mathbf{Z})$ in $H^{1}(G) \approx \operatorname{Hom}(A, \mathbf{Z} / 4 \mathbf{Z})$ under reduction modulo (2), which 
is also the kernel of the mod-2 Bockstein map $\beta_{2}: H^{1}(G) \rightarrow H^{2}(G)$, and so we recover the result of [2]. (This argument shows also that when $G$ is abelian the kernel of cup product with coefficients $\mathbb{F}_{2}$ is isomorphic to the kernel of the mod-2 Bockstein.)

In particular cup product with coefficients $\mathbb{F}_{2}$ is injective if and only if $G_{2} / G_{3}$ is finite of odd order and $G$ does not map onto $\mathbb{Z} / 4 \mathbb{Z}$. For if these conditions hold then $X^{2}(G)=G_{2} X^{4}(G)$ and $G_{2} X^{4}(G) /\left[G, X^{2}(G)\right] X^{4}(G)$ is a quotient of $G_{2} X^{4}(G) / G_{3} X^{4}(G)=0$, since in any case $\left[G, X^{2}(G)\right]$ contains $G_{3}$. Conversely, if cup product is injective then $X^{2}(G)=\left[G, X^{2}(G)\right] X^{4}(G)$, so $G / G_{2} \approx(\mathbb{Z} / 2 \mathbb{Z})^{a} \oplus$ (odd). Since $G_{2} / G_{3}$ is finitely generated and $G_{3} X^{2}\left(G_{2}\right) \subseteq G_{2}$, it shall suffice to assume that $G_{3} X^{2}\left(G_{2}\right)=1$ and show that $G$ must be abelian. But then $G_{2}$ is central and of exponent 2 , so $G$ is a finite nilpotent group. Thus $G \approx T \times$ (odd) where $T$ is a finite group of exponent 4. We may assume $G=T$. But then $X^{2}(G)=\left[G, X^{2}(G)\right] X^{4}(G)=\left[G, X^{2}(G)\right]$ implies that $X^{2}(G)=1$ and so $G$ is abelian. (Note that we have shown that if a 2-group has injective cup product it must be elementary abelian.)

On the other hand let $\theta: F \rightarrow G$ be any map that induces an isomorphism of $F / X^{2}(G)$ with $G / X^{2}(G)$. Then cup product with coefficients $\mathbb{F}_{2}$ is identically 0 if and only if the induced map from $\operatorname{Hom}\left(X^{2}(G) /\left[G, X^{2}(G)\right] X^{4}(G), \mathbb{F}_{2}\right)$ to $\operatorname{Hom}\left(X^{2}(G) /\left[F, X^{2}(F)\right] X^{4}(F), \mathbb{F}_{2}\right)$ is an isomorphism. This is so if and only if the induced map from $F /\left[F, X^{2}(F)\right] X^{4}(F)$ to $G /\left[G, X^{2}(G)\right] X^{4}(F)$ is an isomorphism.

\section{Coefficients $\mathbb{F}_{p}(p$ odd $)$}

Suppose now that $R=\mathbb{F}_{p}$ with $p$ an odd prime. Then $G(R)=G(p)=G_{2}$. $X^{p}(G)$, and $\bar{G} \approx(\mathbf{Z} / p \mathbf{Z})^{r}$ say. We may again use the Künneth theorem to compute $H^{*}(\bar{G})$. Since $H^{*}(\mathbf{Z} / p \mathbf{Z})=\mathbb{F}_{p}[x, y] /\left(x^{2}\right)$ where $x$ generates $H^{1}(\mathbb{Z} / p \mathbb{Z})$ and $y=\beta_{p} x$ is the Bockstein of $x$ in $H^{2}(\mathbb{Z} / p \mathbb{Z})$, the cohomology ring of $\bar{G}$ is the tensor product of an exterior algebra generated in degree 1 by $H^{1}(\bar{G})$ and a polynomial algebra generated in degree 2 by $\beta_{p} H^{1}(\bar{G})$. Explicitly, if $\left\{x_{1}, \ldots, x_{r}\right\}$ is a basis for $H^{1}(\bar{G})$ then $\left\{x_{i} \cup x_{j} \mid 1 \leqslant i<j \leqslant r\right\} \cup\left\{\beta_{p} x_{k} \mid 1 \leqslant k \leqslant r\right\}$ is a basis for $H^{2}(\bar{G})$, and so cup product for $\bar{G}$ is again injective. (In fact it is not hard to show directly, by a calculation with cocycles as in the introductory section of [2], that cup product with coefficients $\mathbb{F}_{p}$ is injective for any abelian group.) Thus we have an exact sequence

$$
0 \rightarrow \operatorname{ker}\left(U_{G}\right) \rightarrow \operatorname{Hom}\left(G(p) /[G, G(p)], \mathbb{F}_{p}\right) \stackrel{\omega}{\rightarrow} \operatorname{coker}\left(U_{\bar{G}}\right) \rightarrow \operatorname{coker}\left(U_{G}\right),
$$

and $\beta_{p} H^{1}(\bar{G})$ maps isomorphically onto $\operatorname{coker}\left(U_{\bar{G}}\right)$. By the naturality of the Bockstein operation, $\beta_{p} H^{1}(\bar{G})$ also maps onto $\beta_{p} H^{1}(G) \subseteq H^{2}(G)$ and the kernel 
of the last map in the above sequence is isomorphic to $\left\{f\right.$ in $H^{1}(G) \mid \beta_{p} f$ is in $\operatorname{Im} U\}$. However any attempt to compute the map $\omega$ seems to lead to cochain calculations even less pleasant than those of [2], and so we shall merely quote from there the result that the kernel of cup product with coefficients $\mathbb{F}_{p}$ is isomorphic to $\operatorname{Hom}\left(G_{2} X^{p}(G) / G_{3} X^{p}(G), \mathbb{F}_{p}\right)=\operatorname{Hom}\left(G(p) / G_{3} X^{p}(G), \mathbb{F}_{p}\right)$, which is easily seen to be naturally a subspace of $\operatorname{Hom}\left(G(p) /[G, G(p)], \mathbb{F}_{p}\right)$. (Curiously, it was the case of $\mathbb{F}_{2}$ rather than that of $\mathbb{F}_{p}$ ( $p$ odd) that seemed the most difficult in [2].)

Thus cup product with coefficients $\mathbb{F}_{p}$ ( $p$ odd) is injective if and only if $G_{2} \subseteq G_{3} X^{P}(G)$. For instance this is so if $G_{2} / G_{3}$ is a finite group of order not divisible by $p$. However the group $P$ presented by $\langle x, y, x| x^{p}=y^{p}=z^{p^{2}}=1$, $\left.[x, y]=z^{p},[x, z]=[x, y]=1\right\rangle$ has injective cup product, although $P_{2} / P_{3} \approx$ $\mathbf{Z} / p \mathbf{Z}$. On the other hand cup product with these coefficients is identically 0 if and only if there is an isomorphism $F / F_{3} X^{p}(F) \approx G / G_{3} X^{p}(G)$.

\section{Acknowledgment}

This note was prompted by an idea of Massey and Traldi [4] who were interested in proving that integral cup product is surjective for the complements of certain unsplittable links.

\section{References}

[1] W. G. Dwyer, 'Homology, Massey products and maps between groups', J. Pure Appl. Algebra 6 (1975), 177-190.

[2] J. A. Hillman, 'The kernel of the cup product', Bull. Austral. Math. Soc. 32 (1985), 261-274.

[3] L. A. Lambe, 'Two exact sequences in rational homotopy theory relating cup products and commutators', Proc. Amer. Math. Soc. 96 (1986), 360-364.

[4] W. S. Massey and L. Traldi, 'On a conjecture of K. Murasugi', Pacific J. Math. 124 (1986), 193-213.

[5] D. Sullivan, 'On the intersection ring of a compact three-manifold', Topology 14 (1975), 275-277.

[6] J. C. Wood, 'A theorem on injectivity of the cup product', Proc. Amer. Math. Soc. 37 (1973), 301-304.

School of Mathematics and Physics

Macquarie University

North Ryde, NSW, 2113

Australia 